\title{
POPDC3 is a potential biomarker for prognosis and radioresistance in patients with head and neck squamous cell carcinoma
}

\author{
XU HE ${ }^{1,2^{*}}$, HONGFA XU ${ }^{2 *}$, WEI ZHAO ${ }^{2}$, MEIXIAO ZHAN ${ }^{2}$, \\ YONG LI ${ }^{2}$, HONGYI LIU ${ }^{2}$, LI TAN ${ }^{3}$ and LIGONG LU ${ }^{1,2}$ \\ ${ }^{1}$ Department of Interventional Oncology, Guangdong Provincial Cardiovascular Institute, Guangdong General Hospital, \\ Guangdong Academy of Medical Sciences, Guangzhou, Guangdong 510080; ${ }^{2}$ Zhuhai Interventional Medical Center, \\ Zhuhai Precision Medical Center, Zhuhai People's Hospital Affiliated with Jinan University, \\ Zhuhai, Guangdong 519000; ${ }^{3}$ Center of Hematology, The First Affiliated Hospital of \\ Guangzhou Medical University, Guangzhou, Guangdong 510230, P.R. China
}

Received December 12, 2018; Accepted August 20, 2019

DOI: $10.3892 / \mathrm{ol} .2019 .10888$

\begin{abstract}
Radiotherapy is the primary means of treatment for patients with head and neck squamous cell carcinoma (HNSCC); however, radioresistance-induced recurrence is the primary cause of HNSCC treatment failure. Therefore, identifying specific predictive biomarkers of the response to radiotherapy may improve prognosis. In the present study, to identify the potential candidate genes associated with radioresistance in patients with HNSCC, the microarray datasets GSE9716, GSE61772 and GSE20549 were downloaded from the Gene Expression Omnibus database. The original CEL files were preprocessed
\end{abstract}

Correspondence to: Professor Ligong Lu, Zhuhai Interventional Medical Center, Zhuhai Precision Medical Center, Zhuhai People's Hospital Affiliated with Jinan University, 95 Kangning Road, Zhuhai, Guangdong 519000, P.R. China

E-mail: luligong1969@126.com

Professor Li Tan, Center of Hematology, The First Affiliated Hospital of Guangzhou Medical University, 1 Kangda Road, Guangzhou, Guangdong 510230, P.R. China

E-mail: tanya_tanli@163.com

*Contributed equally

Abbreviations: HNSCC, head and neck squamous cell carcinoma; GEO, Gene Expression Omnibus; FC, fold-change; DEGs, differentially expressed genes; DAVID, Database for Annotation Visualization and Integrated Discovery; GO, Gene Ontology; KEGG, The Cancer Genome Atlas; PPI, protein-protein interaction; WGCNA, Weighted Correlation Network Analysis; MM, module membership; GS, gene significance; POPDC3, popeye domain-containing protein 3; OS, overall survival

Key words: bioinformatics analysis, head and neck cancer, weighted correlation network analysis, popeye domain-containing protein 3, radiation resistance using the Affymetrix package and quantile normalization and background correction were conducted using the Core package in Bioconductor. The GSE9716 dataset, consisting of 18 irradiated and 16 non-irradiated samples, was divided into two groups according to their exposure to irradiation: i) Non-irradiation group, which included 8 radioresistant samples and 8 radiosensitive samples; and ii) post-irradiation group, which included 9 radioresistant samples and 9 radiosensitive samples. The two groups were treated as separate datasets and screened. A total of 86 differentially expressed genes (DEGs) were identified in the non-irradiation group and 405 DEGs in the post-irradiation group. Gene Ontology enrichment and Kyoto Encyclopedia of Genes and Genomes pathway analysis detected several significant functions associated with the DEGs. In the co-expression analysis, 76 hub genes in the light green module and 917 hub genes with a high connectivity were selected for further analysis. Finally, overlapping the DEGs and hub genes from the two groups yielded a map of 13 shared differentially expressed genes. The 13 genes showed significantly different expression in radioresistant samples compared with the radiosensitive samples before and after irradiation. Out of these genes, popeye domain-containing protein 3 (POPDC3) was highly expressed in the post-irradiation group compared with the non-irradiation group. In survival analysis, high POPDC3 expression correlated with poor a prognosis for patients with HNSCC. The independent prognostic factors were identified using univariate and multivariate Cox analyses based on The Cancer Genome Atlas database. These were incorporated into a nomogram to predict 3- and 5-year overall survival. Receiver operating characteristic curves were used to estimate the accuracy of the nomogram. Together these studies suggest that POPDC3 may serve as a potential predictive biomarker for prognosis and radioresistance of patients with HNSCC as well as clinical diagnosis and treatment of patients.

\section{Introduction}

Head and neck squamous cell carcinoma (HNSCC), including nasopharyngeal cancer, hypopharyngeal cancer, laryngeal 
cancer and tonsillar cancer, consists of different pathological and molecular subtypes, with differing metastatic potentials and prognoses. HNSCC is the sixth most common cancer worldwide, with $>600,000$ new cases diagnosed each year (1). The treatment of HNSCC is based on a combination of chemotherapy, radiotherapy and surgery or chemoradiation alone. These conventional treatments have several limitations. Surgery may cause secondary injury and reduce the patients' quality of life. Radiotherapy is one of the most effective treatment options against cancer. The development of anatomical personalization has allowed radiation oncologists to improve the outcomes of numerous patients with HNSCC. However, the efficacy of radiation therapy is often impeded by tumor radioresistance, resulting in treatment failure and tumor relapse. Currently, there are few biomarkers available in the clinical setting for predicting tumor radiotherapy outcome in patients with HNSCC (2). There is still an urgent need to identify effective biomarkers of radioresistance to guide individualized treatment.

The development of microarray technologies provides an ideal tool for biomarker screening. Numerous gene sequences have been stored in the Gene Expression Omnibus (GEO) database (3), which may provide crucial data for bioinformatic mining and acquisition of data from multiple samples. Using integrative analysis, the publicly available GEO database may be used to identify key genes and corresponding pathways as well as the interactive network, therefore providing deeper insight into the underlying molecular mechanisms $(4,5)$. However, at present there have been few studies that have utilized integrative analysis of GEO datasets to identify radioresistance-associated genes in patients with HNSCC. In the present study, through comprehensive analysis of the GEO datasets, a set of differentially expressed genes (DEGs) were identified, which are potentially involved in radioresistance and pathological progression of HNSCC.

The identified genes have rarely been reported as prognostic biomarkers for HNSCC. Additionally, as a public dataset was used to identify the prognostic biomarkers associated with radiation resistance in patients with HNSCC, these biomarkers have not been conclusively shown to be associated with HNSCC radioresistance. Genome-wide association studies of patients with HNSCC with differing sensitivities to radiation combined with combinatorial bioinformatics may supply a framework for further identifying the expression profiles which may predict the response to radiotherapy. Furthermore, network-based analysis programs may be employed to validate key-regulator transcriptional profiles for the prediction of radiotherapy response. In the present study, the GSE9716 dataset was divided into two groups according to the exposure to irradiation: i) The non-irradiation group ( 8 radioresistant samples and 8 radiosensitive samples); and ii) the post-irradiation group ( 9 radioresistant samples and 9 radiosensitive samples). A total of 86 DEGs were identified in the non-irradiation group and 405 DEGs in the post-irradiation group. Functional pathway analysis was performed, including Gene Ontology (GO) term enrichment analysis and Kyoto Encyclopedia of Genes and Genomes (KEGG) pathway analysis using the Database for Annotation Visualization and Integrated Discovery (DAVID) (6-8). Weighted Correlation Network Analysis (WGCNA) was performed to identify the hub genes in each group (9). A total of 13 genes were found to show significantly different expression in the radioresistant samples compared with the radiosensitive samples prior to and following irradiation. According to the fold-change of gene expression, Kaplan-Meier analysis and protein-protein interaction analysis in the post-irradiation group compared with the non-irradiation group, it was demonstrated, for the first time to the best of our knowledge, that a high level of POPDC3 expression may contribute to radioresistance in patients with HNSCC and that POPDC3 may be a potential biomarker for predicting radiosensitivity and prognosis in patients with HNSCC, The prognostic value of POPDC3 in The Cancer Genome Atlas (TCGA) cohort was determined using X-tile analysis. A total of 161 patients with HNSCC were included and the clinicopathological features were analyzed with respect to occurrence of HNSCC. The clinical diagnostic significance of POPDC3 in patients with HNSCC was comprehensively evaluated using $\mathrm{X}$-tile and nomogram analysis.

\section{Materials and methods}

Microarray data. The microarray expression profile datasets GSE9716 (10), GSE61772 (11) and GSE20549 were downloaded from the GEO database (https://www.ncbi.nlm.nih. gov/geo/). The datasets were based on the GPL96 [HG-U133A] Affymetrix Human Genome U133A Array, GPL6884 Illumina HumanWG-6 version 3.0 expression beadchip and GPL6244 [HuGene-1_0-st] Affymetrix Human Gene 1.0 ST Array platforms, respectively. In the GSE9716 dataset, there were 38 different treated HNSCC samples derived from the parental radiosensitive tumor SCC-61 and radioresistant tumor nu61 cell lines and xenografts (18 samples were irradiated at 3 Gy and 16 samples were left untreated, four samples treated with interferon were removed). In GSE61772, six samples were included with three radiosensitive esophageal cancer cell lines and three radioresistant counterparts. For GSE20549, there were 21 radioresistant H1299 lung cancer cell lines and 21 radiosensitive $\mathrm{H} 460$ lung cancer cell lines.

Data preprocessing. The original CEL files were preprocessed with the robust multiarray average function in the Affymetrix package in $\mathrm{R}$ language ( $\mathrm{R}$ version 3.3 .5 ) (12). The quality of these datasets was assessed with the affyPLM package. The RNA degradation images, FitPLM weight, residual relative log expression and normalized unscaled standard errors of the CEL data were evaluated. The Core package in Bioconductor (13) and the limma R package (14) were used for background correction and quantile normalization.

After data preprocessing, the GSE9716 dataset was divided into two groups i) the non-irradiation group which had 8 radioresistant samples and 8 radiosensitive samples; and ii) the post-irradiation group which consisted of 9 radioresistant samples and 9 radiosensitive samples treated with 3 Gy irradiation. These two groups were used for all subsequent analysis.

Identification of DEGs. DEGs between the two groups were selected and identified based on a Student's t-test analysis of linear models for the limma package in R. Fold-change (FC) of gene expression was calculated with a cut-off value of 
$\mid \log 2 \mathrm{FCl} \geq 1.0$ and a P-value $<0.01$ for DEG selection. Funrich (version 3.1.3; funrich.org/) was used to analyze the overlapping profiles of DEGs among the different datasets.

Functional network enrichment analyses of candidate genes. The Database for Annotation, Visualization and Integrated Discovery (DAVID; http://david.ncifcrf.gov), an online biological information database, provides a comprehensive set of annotated functional information on genes and proteins. To analyze the function of DEGs, GO and KEGG pathway enrichment analysis were performed using the DAVID online database. The Search Tool for the Retrieval of Interacting Genes database (string-db.org/) was used to construct a protein-protein interaction (PPI) network. Cytoscape (cytoscape.org) was used to evaluate the interactive correlation between different proteins. Oncomine (oncomine. org/), firebrowse (firebrowse.org/) and protein atlas database (proteinatlas.org/) were used to validate the expression of POPDC3 in HNSCC.

Co-expression network and identification of hub genes. A WGCNA package was used to evaluate the correlation of DEGs of the two groups and to search for the most significant relevant gene modules associated with radioresistance (15). The soft thresholding power of the non-irradiation group was set as 20 and the post-irradiation group was set as 22 to certify a scale-free network. The genes that had the soft-thresholding power below 20 in non-irradiation group or 22 in post-irradiation groups were defined as weak correlations and thus were merged. A total of eight modules were recognized in the non-irradiation group and a total of four modules in the post-irradiation group. Module membership (MM) and gene significance (GS) were used to analyze the correlation between each module and radioresistance. Several modules showed an association with radioresistance in the two groups and only the correlation between the light green module in the non-irradiation group $\left(\mathrm{P}<0.01, \mathrm{R}^{2}=0.98\right)$ and the blue module in the post-irradiation with radioresistance was high $\left(\mathrm{P}<0.01, \mathrm{R}^{2}=0.99\right)$. The two modules were highly correlated with radioresistance and were thus used for subsequent analysis (Figs. 2B and 3B). Hub genes play a significant role in cell biological processes $(16,17)$. Genes with a high MM were set as candidate hub genes for radioresistance in the module, with weighted correlation coefficients $>0.8$ (Figs. 2C and 3C).

Statistical analysis. Statistical analyses were performed using SPSS version 17 (SPSS, Inc.). The key genes were analyzed using Kaplan Meier-Plotter (http://kmplot.com/analysis/), an online database for survival data analyses. HNSCC samples were split into two groups as follows based on POPDC3 expression (high vs. low/medium). To determine whether the predictive power of POPDC 3 expression level could be independent of other clinical variables. The univariate and multivariate cox regression analyses, as well as Kaplan-Meier curves were conducted, hazard ratios and corresponding 95\% confidence interval (CI) of variables were also calculated. Then a prognostic nomogram model was constructed to further evaluate the prognostic ability of the marker. The appropriate cut-off values for POPDC 3 expression level based on OS information were determined with X-tile software
(Yale University; version 3.6.1). All data are presented as the mean \pm standard error of the mean. $\mathrm{P}<0.05$ was considered to indicate a statistically significant difference.

\section{Results}

Identification of DEGs in head and neck cancer. After data preprocessing, the GSE9716 dataset was divided into two groups according to their exposure to irradiation: Non-irradiation group and post-irradiation group. The DEGs of each group were analyzed based on the limma $\mathrm{R}$ package. $\mathrm{P}<0.05$ and $\mid \log 2 \mathrm{FCl}>1$ were set as the cut-off values. Based on the cut-off value, a total of 86 DEGs were validated in the non-irradiation group, consisting of 77 upregulated genes and 9 downregulated genes. A total of 405 DEGs were validated in the post-irradiation group, consisting of 385 upregulated genes and 20 downregulated genes (Fig. 1A and C). The significantly DEGs are presented in Fig. 1B and D.

KEGG and GO enrichment analyses of DEGs. GO enrichment profiles and KEGG pathways were generated from the differentially expressed genes in the two groups, and the results are summarized in Tables I and II. The interferon- $v$-mediated signaling pathway (GO:0060333), the response to stilbenoid (GO:0035634), leukocyte cell-cell adhesion (GO:0007159) and others were enriched in the non-irradiation group (Table I). Viral carcinogenesis, activation of mitogen associated protein kinase (MAPK) activity, positive regulation of $\kappa \mathrm{B}$ kinase/NF- $\kappa \mathrm{B}$, complement and coagulation cascades and others were significantly enriched in the post-irradiation group. These results suggest that the biologically active signaling pathways were notably different prior to and following irradiation. The significantly enriched functions may improve understanding of the characteristics and functions of DEGs involved in HNSCC.

Co-expression network construction and hub module identification. Using the WGCNA package in R, the genes in each group with highly similar expression patterns were combined into the modules using average linkage hierarchical clustering. A power 20 for the non-irradiation group and 22 for the post-irradiation group were set as the soft-thresholding to certify a scale-free network. In the non-irradiation group, a total of eight modules were recognized (Fig. 2A), whereas in the post-irradiation group, four modules were identified (Fig. 3A). The association between each module and radioresistance traits were demonstrated in each group (Figs. 2B and 3B). The association between the light green module and radioresistance traits was high $\left(\mathrm{P}=1.9 \times 10^{-20}, \mathrm{R}^{2}=0.83\right)$ in the non-irradiation group (Fig. 2B), whereas in the post-irradiation group, the blue module was highly associated with radioresistance traits $\left(\mathrm{P}=1 \times 10^{-200}, \mathrm{R}^{2}=0.84\right.$; Fig. 3B). The corresponding heatmaps of all genes are shown in Figs. 2C and 3C. Furthermore, a scatter diagram of the correlation between GS for radioresistance traits and MM in the light green or blue module are shown in each group in Figs. 2D and 3D, respectively, which exhibited an extremely strong association with HNSCC. There were reported hub genes, which served notable functions in various cell processes $(7,8)$. Weighted correlation coefficients $>0.8$ were set for the candidate hub genes for radioresistance in the module. A total of 76 genes in the light green module and 


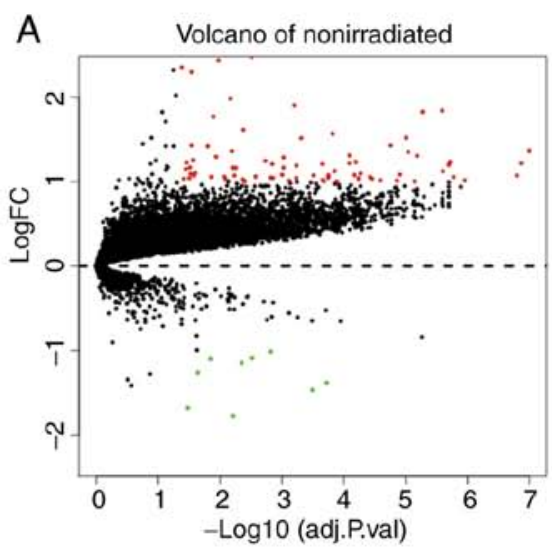

B
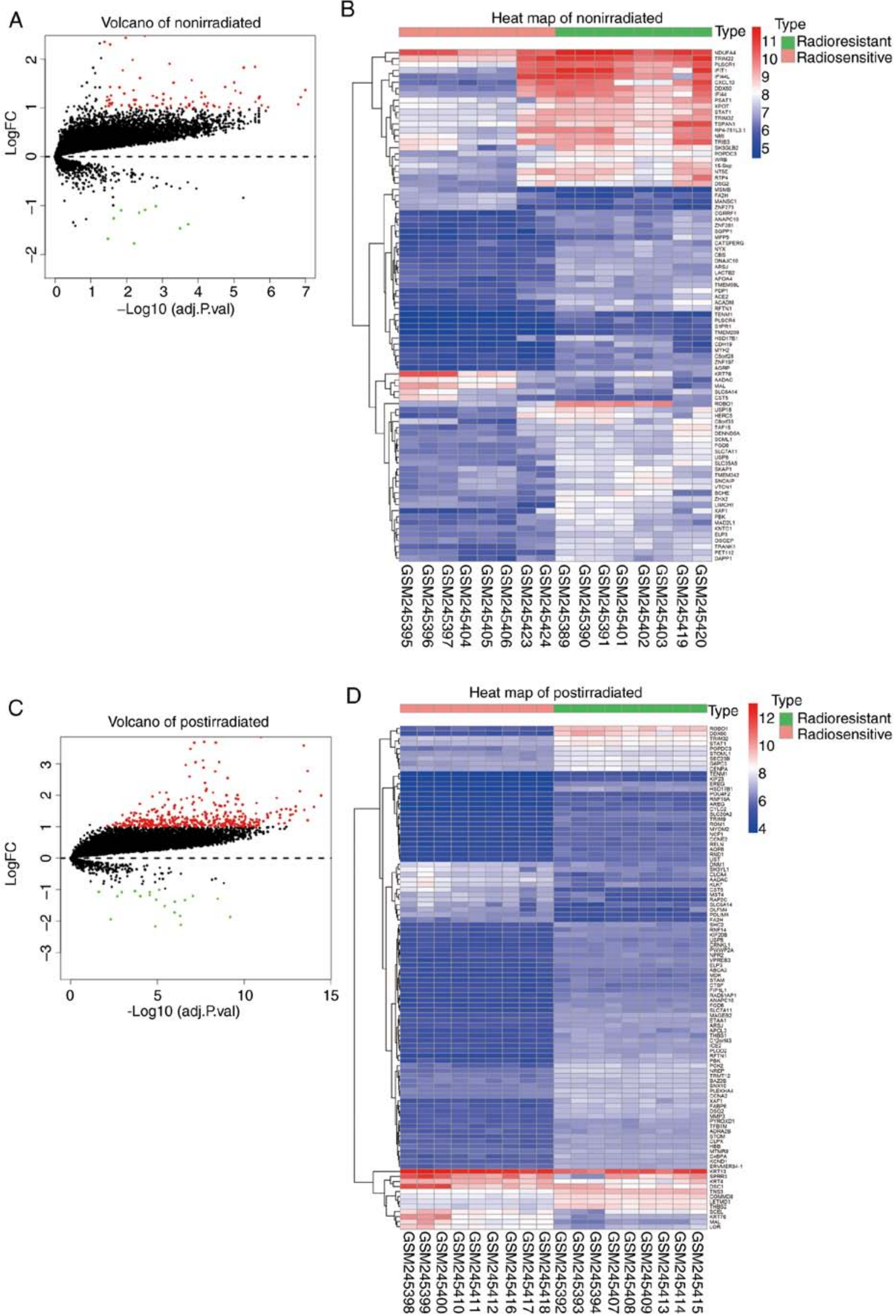

Figure 1. DEGs identified in GSE9716. (A) Volcano plot of the DEGs in the non-irradiation group in radiosensitive and radioresistant samples. (B) Hierarchical clustering dendrograms of the DEGs according to the value of $\log 2 \mathrm{FCl}$ in the non-irradiation group. (C) Volcano plot of the DEGs in the post-irradiation group in radiosensitive and radioresistant samples. (D) Hierarchical clustering dendrograms of the DEGs according to the value of $1 \log 2 \mathrm{FCl}$ in the post-irradiation group. In the volcano plots, red dots represented upregulated genes and green dots represented downregulated genes. DEG, differentially expressed gene; FC, fold-change. 
Table I. GO enrichment analysis of differentially expressed genes of nonirradiated group.

\begin{tabular}{llrr}
\hline Term & \multicolumn{1}{c}{ Description } & Count in gene set & P-value \\
\hline GO:0060333 & Interferon- $\boldsymbol{\gamma}$-mediated signaling pathway & 2 & 0.0129 \\
GO:0035634 & Response to stilbenoid & 2 & 0.0213 \\
GO:0010898 & Positive regulation of triglyceride catabolic process & 2 & 0.0255 \\
GO:0032287 & Peripheral nervous system myelin maintenance & 2 & 0.0297 \\
GO:0051607 & Defense response to virus & 3 & 0.0413 \\
GO:0007159 & Leukocyte cell-cell adhesion & 2 & 0.0422 \\
\hline
\end{tabular}

GO, gene ontology.

Table II. GO and Kyoto Encyclopedia of Genes and Genomes pathway enrichment analysis of differentially expressed genes of post-irradiated group.

\begin{tabular}{|c|c|c|c|}
\hline Term & Description & Count in gene set & P-value \\
\hline GO:0060337 & Type I interferon signaling pathway & 27 & $1.17 \times 10^{-26}$ \\
\hline GO:0051607 & Defense response to virus & 25 & $2.65 \times 10^{-13}$ \\
\hline GO:0060333 & Interferon- $\gamma$-mediated signaling pathway & 17 & $2.69 \times 10^{-12}$ \\
\hline GO:0045087 & Innate immune response & 23 & $2.61 \times 10^{-04}$ \\
\hline GO:0016032 & Viral process & 17 & 0.0011 \\
\hline GO:0043123 & Positive regulation of $\mathrm{I}-\kappa \mathrm{B}$ kinase/NF- $\kappa \mathrm{B}$ signaling & 11 & 0.0032 \\
\hline GO:0051603 & Proteolysis involved in cellular protein catabolic process & 6 & 0.0041 \\
\hline GO:0032728 & Positive regulation of interferon- $\beta$ production & 4 & 0.0214 \\
\hline GO:0000187 & Activation of MAPK activity & 7 & 0.0321 \\
\hline GO:0001916 & Positive regulation of $\mathrm{T}$ cell mediated cytotoxicity & 3 & 0.0327 \\
\hline hsa05168 & Herpes simplex infection & 17 & $1.70 \times 10^{-05}$ \\
\hline hsa04145 & Phagosome & 14 & $1.18 \times 10^{-04}$ \\
\hline hsa05169 & Epstein-Barr virus infection & 10 & 0.0041 \\
\hline hsa05203 & Viral carcinogenesis & 13 & 0.0064 \\
\hline hsa04913 & Ovarian steroidogenesis & 6 & 0.0081 \\
\hline hsa04610 & Complement and coagulation cascades & 7 & 0.0084 \\
\hline hsa05332 & Graft-versus-host disease & 5 & 0.0097 \\
\hline hsa04612 & Antigen processing and presentation & 7 & 0.0132 \\
\hline hsa05330 & Allograft rejection & 5 & 0.0144 \\
\hline hsa04144 & Endocytosis & 13 & 0.0213 \\
\hline
\end{tabular}

GO, gene ontology; MAPK, mitogen-activated protein kinase; NF, nuclear factor.

917 genes in the blue module with a high connectivity were selected for further analysis.

Selection of POPDC3 and its utility as a biomarker of radioresistance in patients with HNSCC. The overlap among DEGs from the two groups and the hub genes of the light green and blue modules, contained 13 shared DEGs: RFTN1, PBK, TENM1, ARSJ, ELP3, USP8, XAF1, POPDC3, LACTB2, ZHX2, PET112, TRANK1 and ACADM (Fig. 4A). The 13 genes were screened as candidate genes for their ability to predict radioresistance in patients with HNSCC, which may serve to maintain the radioresistance profile of cancer cells and promote the radioresistant abilities of cancer cells following irradiation. The FC of expression of the 13 candidate genes in the post-irradiation group relative to the non-irradiation group is shown in Fig. 4B. PBK and POPDC3 were the top two high expression genes in the post-irradiation group compared with the non-irradiation group (Fig. 4B). The effects of these two genes on the survival of patients with HNSCC was evaluated by Kaplan-Meier analysis. Only POPDC3 was significantly associated with the prognosis of patients with HNSCC $(\mathrm{P}<0.001)$. The overall survival (OS) was significantly longer in patients with lower levels of POPDC3 expression compared with patients with higher levels of POPDC3 expression $(\mathrm{P}<0.001$; Fig. 4C). In addition, several datasets were used to identify the mRNA expression levels of POPDC3. The results further demonstrated that POPDC3 expression was significantly increased in HNSCC tissues compared with normal tissues 
A

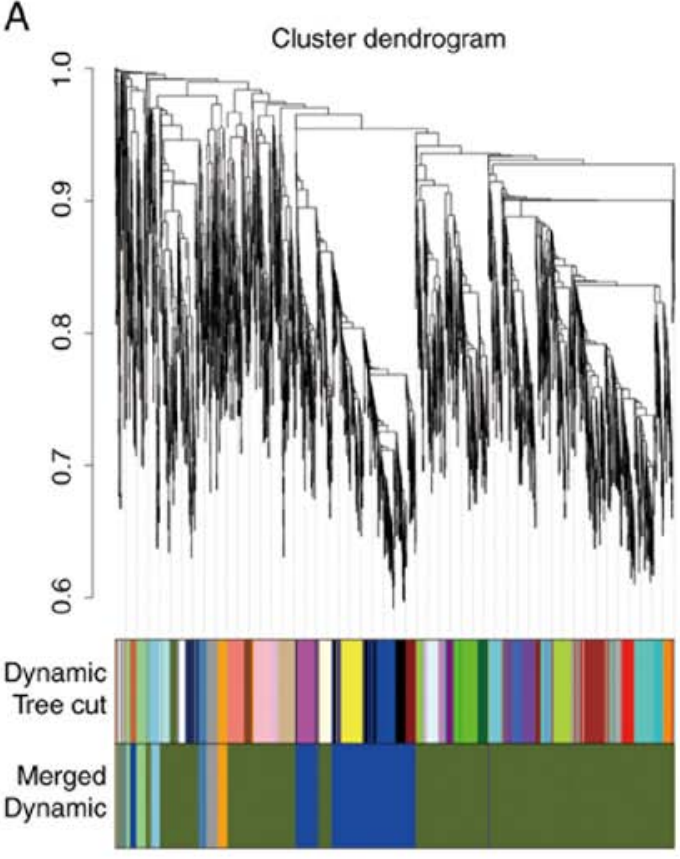

C

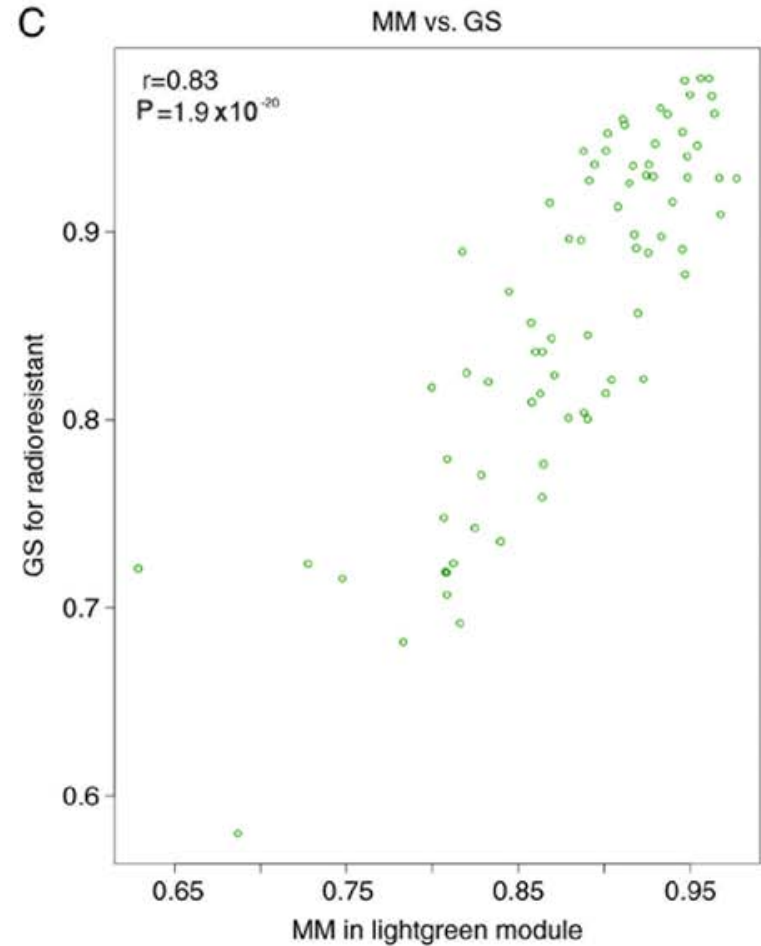

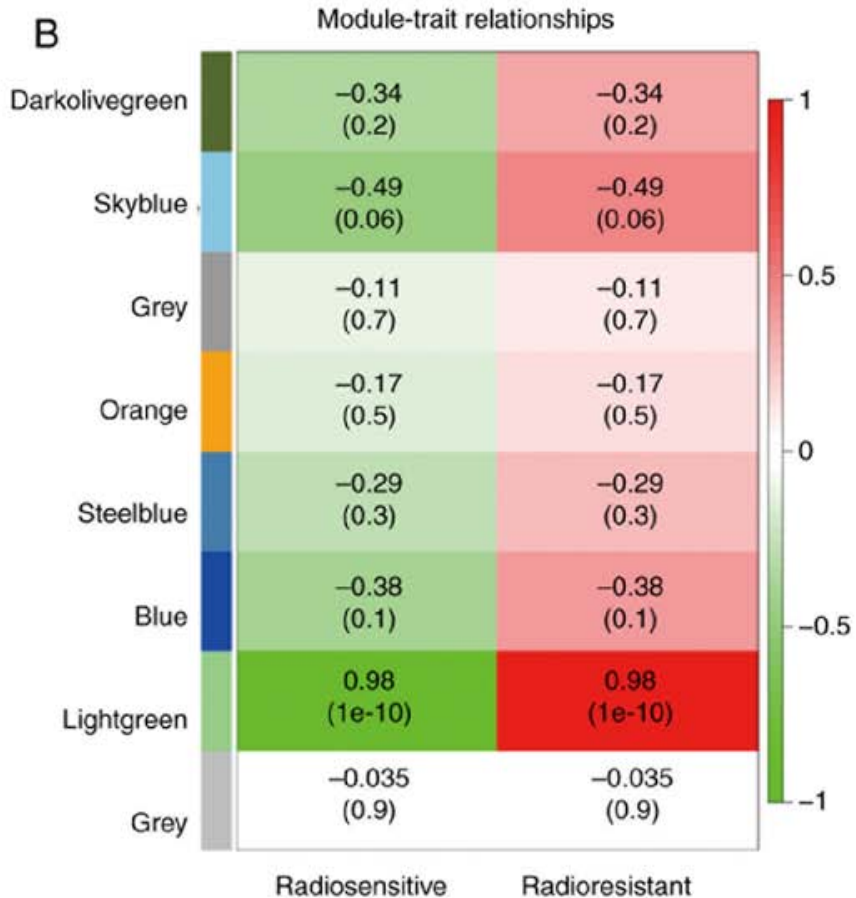

D

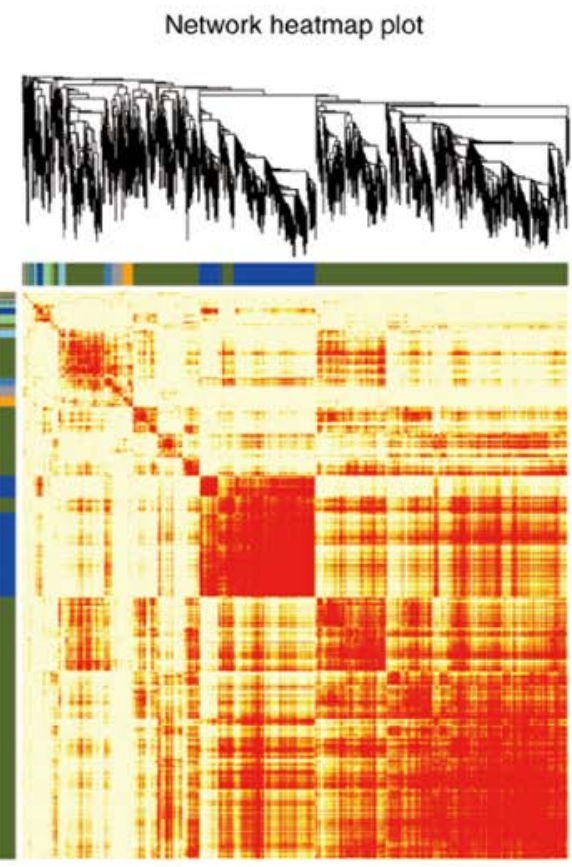

Figure 2. Identification of modules associated with radioresistance in the non-irradiation group. (A) Hierarchical cluster tree shows the co-expression modules of all the DEGs identified by WGCNA. The top panel shows a gene dendrogram and the bottom panel shows the gene modules with different colors. The panels were merged to form dynamic modules labeled with different colors and gene correlation. (B) Correlation between modules and clinical traits. Each cell refers to the corresponding correlation and P-value. The light green modules were most probably associated with radioresistance traits. (C) A scatter map of gene significance for radioresistance traits vs. the module membership in the light green module. Genes in the module were highly associated with radioresistance traits $\left(\mathrm{P}=1.9 \times 10^{-20}, \mathrm{R}^{2}=0.83\right)$. (D) Network heatmap plot of all the DEGs. Each branch of the hierarchical clustering dendrograms refers to one of the eight modules. The color bars between the dendrograms and the heatmap plot represent the color-coded eigengene-based connectivity. The intensity of the red color indicates highly interconnected genes in each module on a linear scale. DEG, differentially expressed gene; WGCNA, weighted correlation network analysis; MM, Module membership; GS, gene significance.

(P<0.05; Fig. 4D-G). Based on raw data mining from the GEO database (GSE61772 and GSE20549), POPDC3 expression was significantly increased in the radioresistant samples compared with the radiosensitive samples in esophageal and lung cancer
$(\mathrm{P}<0.01$; Fig. $4 \mathrm{H}$ and $\mathrm{I})$, further illustrating the value of POPDC 3 expression as a biomarker for radioresistance and the role it may serve in various types of cancer. Finally, a PPI network based on POPDC 3 was constructed. Several proteins 


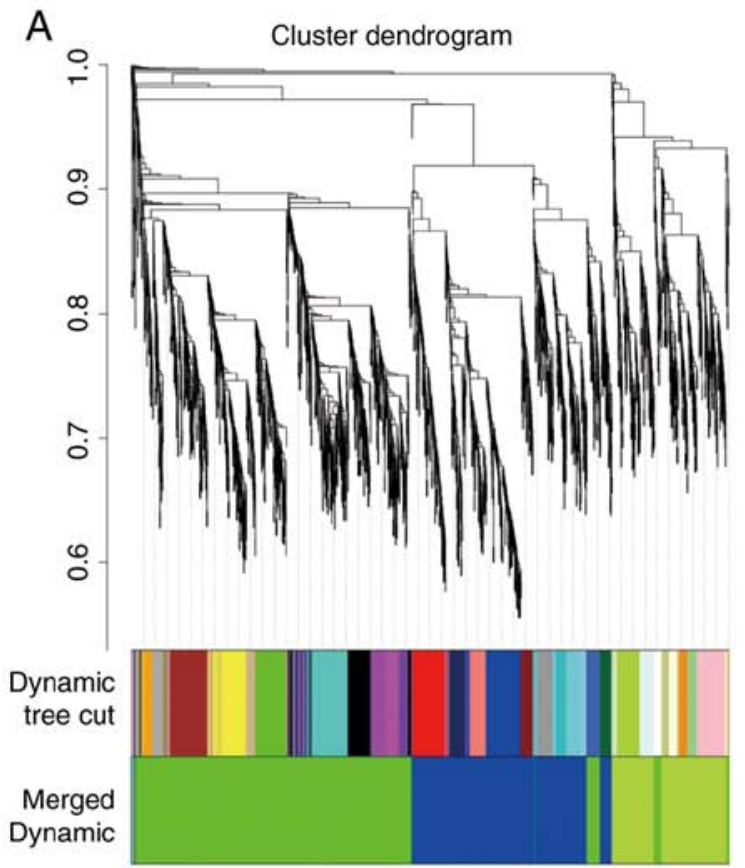

C

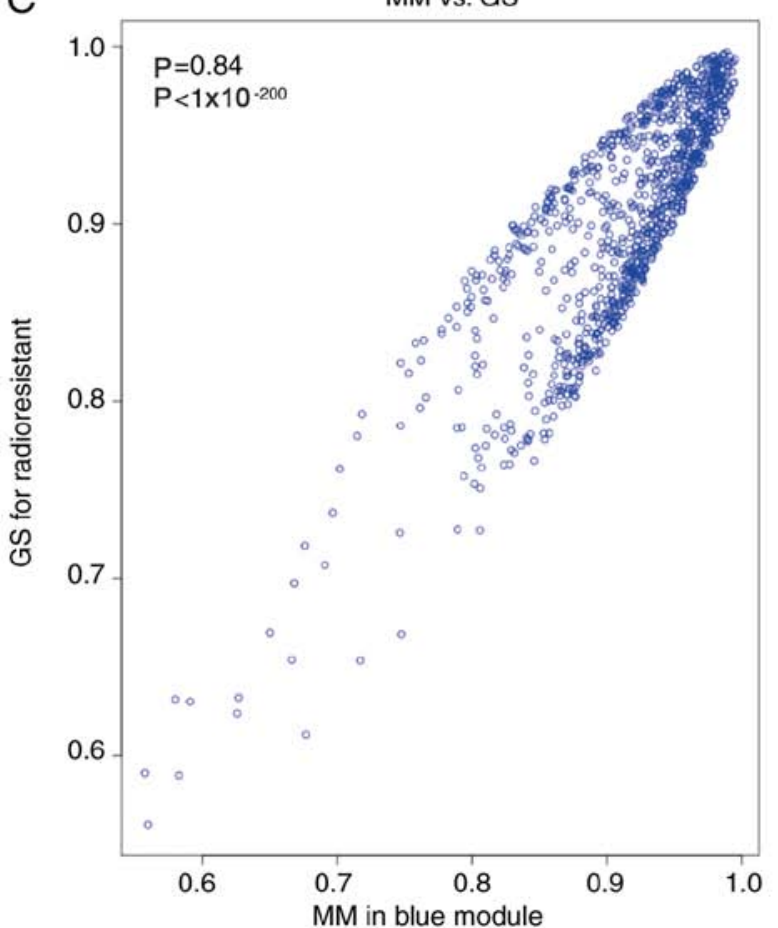

B

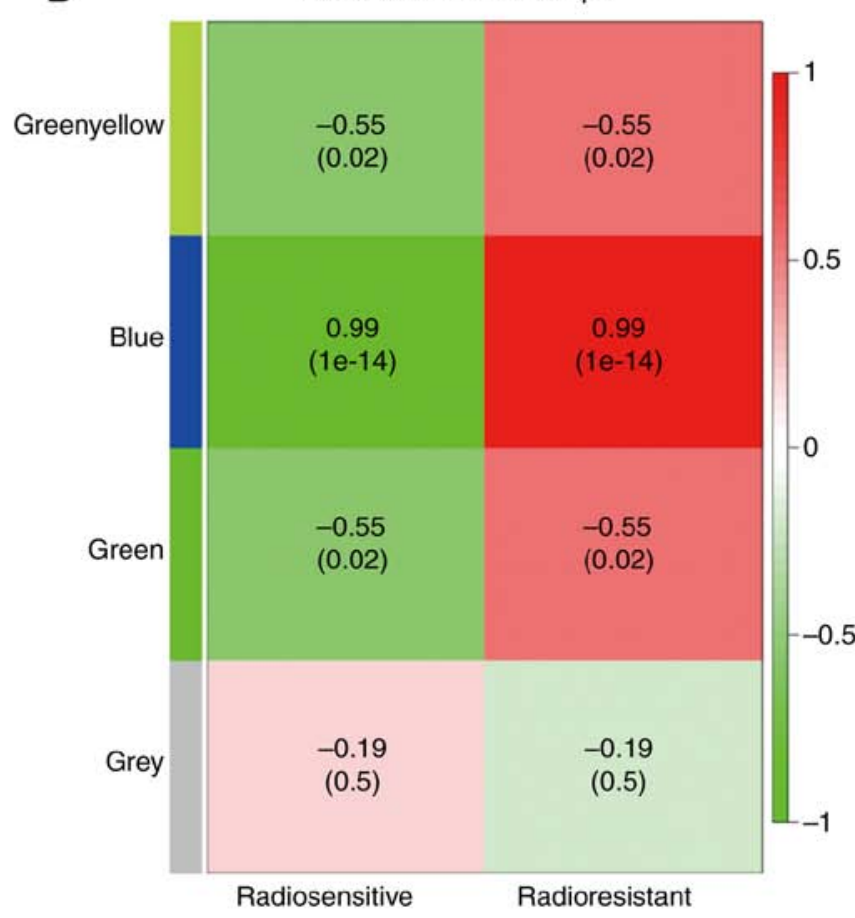

D
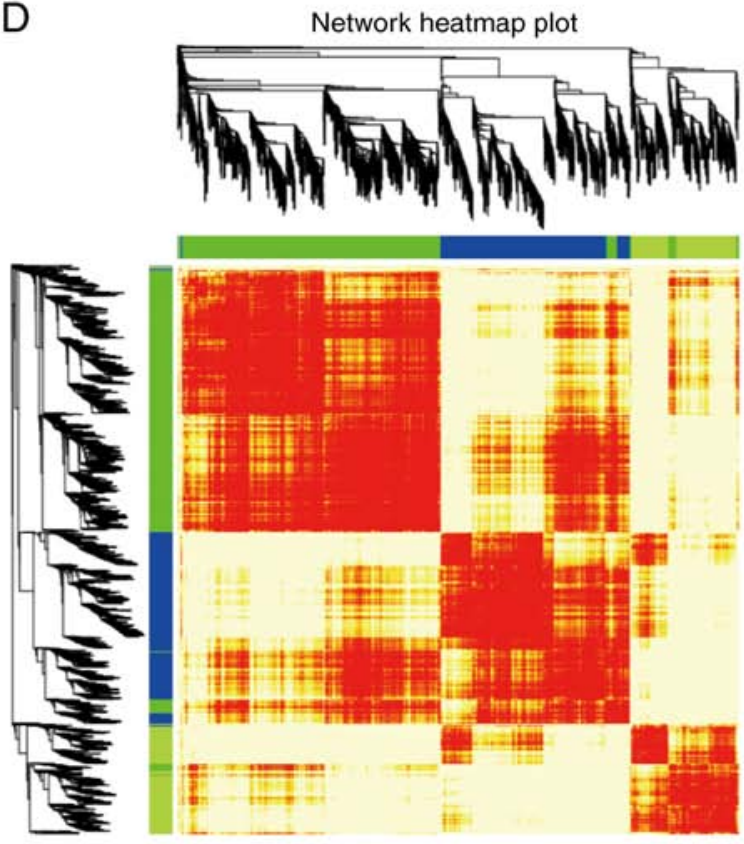

Figure 3. Identification of modules associated with radioresistance in the post-irradiation group. (A) Hierarchical cluster tree showing the co-expression modules of all the DEGs identified by weighted gene correlation network analysis. (B) Correlation between modules and clinical traits. The blue modules indicate the modules that were best correlated with radioresistance traits. (C) A scatter map of gene significance for radioresistance traits vs. module membership in the blue module. Genes in the module were highly significantly correlated with radioresistance traits $\left(\mathrm{P}=1 \times 10^{-200}, \mathrm{R}^{2}=0.84\right)$. (D) Network heatmap plot of all DEGs. Each branch of the hierarchical clustering dendrograms refers to each of the 4 modules. The color bars between the dendrograms and the heatmap plot represent the color-coded eigengene-based connectivity. The intensity of the red color indicates highly interconnected genes in each module on a linear scale. DEG, differentially expressed gene; MM, Module membership; GS, gene significance.

participating in the progression of cancer, such as KIF20A and SLU7 interacted with POPDC3 according to the PPI network.

Prognostic variables. Data on 161 patients with HNSCC was obtained from TCGA (Table III). The survival times of the patients with HNSCC in TCGA was used to perform X-tile analysis using $\mathrm{X}$-tile. The analyses showed that high expression of POPDC 3 was associated with poor clinical outcome compared with patients with low and medium levels of POPDC 3 expression. The X-tile analysis with the optimal 
A

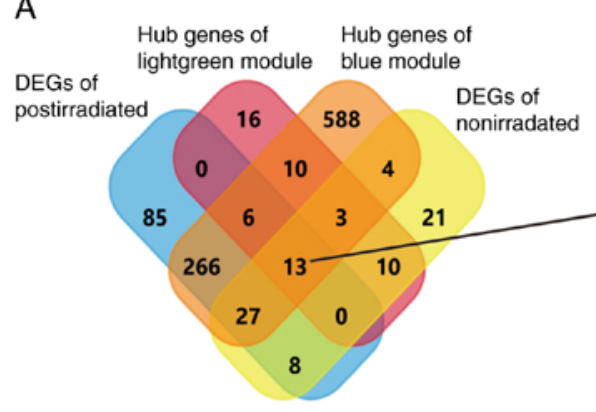

B

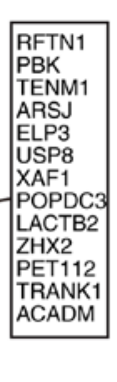

C PBK
POPDC3
TENM1
ARSJ
RFTN1
ELP3
LACTB2
XAF1
PET112
USP8
TRANK1
ZHX2
ACADM

C POPDC3

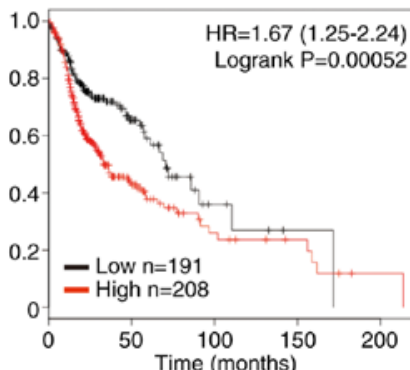

D
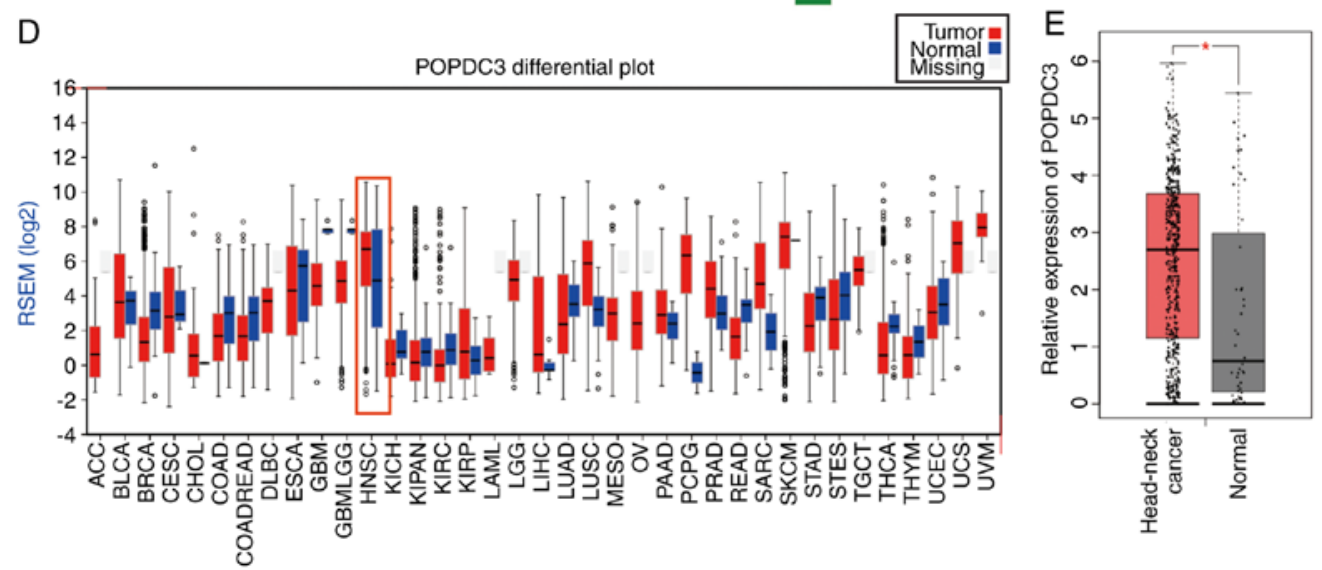

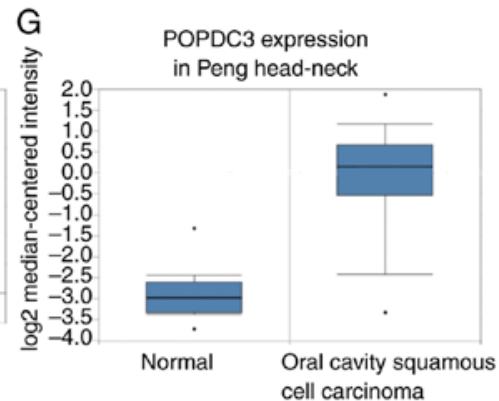

cell carcinoma
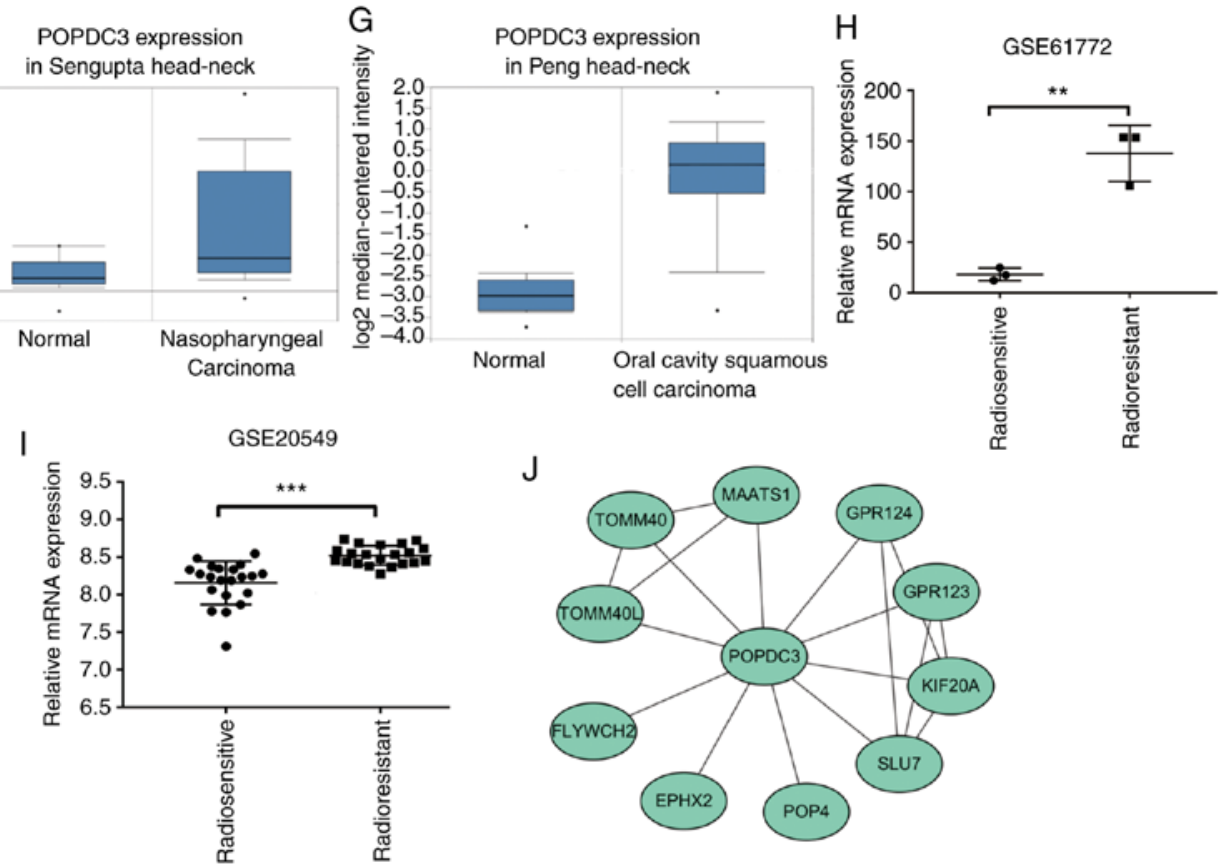

$J$

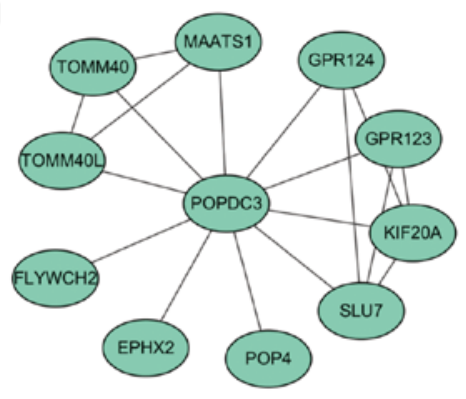

Figure 4. POPDC3 expression profile in different public datasets and utility as a biomarker and prognostic factor for radioresistance in patients with HNSCC. (A) Venn diagram of the differentially expressed genes of the post-irradiation, non-irradiation and hub genes in the blue module, and hub genes in the light green module identified 13 candidate genes. (B) Gene expression profiling was performed on the post-irradiation and non-irradiation groups. Red and green represent the fold-change of expression of the 13 candidate genes in the post-irradiation group relative to the non-irradiation group, red represented upregulated genes and green represented downregulated genes. (C) Kaplan-Meier analysis indicated that the patients with HNSCC with a high expression level of POPDC3 have a poor overall survival compared patients with a low expression level of POPDC3 ( $\mathrm{P}=0.00052$ ). A high level of expression of POPDC3 in HNSCC compared with normal tissues in the (D) firebrowse database, (E) protein atlas database and Oncomine database in (F) nasopharyngeal carcinoma and (G) oral cavity squamous cell carcinoma. Validation of POPDC3 mRNA expression levels according to the Gene Expression Omnibus databases (H) GSE61772 and (I) GSE20549. A total of two of the datasets showed a higher level of expression of POPDC3 in radioresistant samples compared with the counterpart radiosensitive samples. (J) Protein-protein interaction network of POPDC3. Several genes participating in cancer progression, such as KIF20A and SLU7, linearly interacted with POPDC3. HNSCC, head and neck squamous cell carcinoma; POPDC3, popeye domain-containing protein $3 .{ }^{*} \mathrm{P}<0.05,{ }^{* *} \mathrm{P}<0.01,{ }^{* * *} \mathrm{P}<0.001$. 
Table III. Association of POPDC3 expression with clinicopathological characteristics in head and neck squamous cell carcinoma patients.

\begin{tabular}{|c|c|c|c|c|c|}
\hline \multirow[b]{2}{*}{ Characteristics } & \multicolumn{2}{|c|}{ Patients } & \multicolumn{2}{|c|}{$\begin{array}{l}\text { POPDC3 } \\
\text { expression }\end{array}$} & \multirow[b]{2}{*}{ P-value } \\
\hline & $\mathrm{n}$ & $\%$ & High & Low & \\
\hline All patients & 161 & 100 & 80 & 81 & \\
\hline Sex & & & & & $<0.001^{\mathrm{a}}$ \\
\hline Male & 124 & 77.0 & 58 & 66 & \\
\hline Female & 37 & 23.0 & 31 & 6 & \\
\hline Age (years) & & & & & $<0.001^{\mathrm{a}}$ \\
\hline$<60$ & 69 & 42.9 & 33 & 36 & \\
\hline$\geq 60$ & 92 & 57.1 & 71 & 21 & \\
\hline T_stage & & & & & 0.600 \\
\hline $\mathrm{T} 1$ & 7 & 4.3 & 3 & 4 & \\
\hline $\mathrm{T} 2$ & 35 & 21.7 & 3 & 4 & \\
\hline $\mathrm{T} 3$ & 35 & 21.7 & 15 & 20 & \\
\hline $\mathrm{T} 4$ & 84 & 52.3 & 46 & 38 & \\
\hline N_stage & & & & & 0.244 \\
\hline No & 74 & 46.0 & 32 & 42 & \\
\hline N1 & 32 & 21.7 & 16 & 16 & \\
\hline $\mathrm{N} 2+\mathrm{N} 3$ & 55 & 32.3 & 32 & 23 & \\
\hline Grade & & & & & 0.070 \\
\hline 1 & 17 & 10.6 & 4 & 13 & \\
\hline 2 & 102 & 63.4 & 53 & 49 & \\
\hline 3 & 42 & 26.1 & 23 & 19 & \\
\hline Clinical_stage & & & & & 0.347 \\
\hline $\mathrm{I}+\mathrm{II}$ & 24 & 14.9 & 10 & 14 & \\
\hline III & 29 & 18.0 & 12 & 14 & \\
\hline IV & 108 & 67.1 & 58 & 50 & \\
\hline LN_positive & & & & & 0.062 \\
\hline Negative & 64 & 39.8 & 26 & 38 & \\
\hline Positive & 97 & 60.2 & 54 & 43 & \\
\hline LN_invasion & & & & & 0.794 \\
\hline Negative & 99 & 61.5 & 50 & 49 & \\
\hline Positive & 62 & 38.5 & 30 & 32 & \\
\hline
\end{tabular}

astatistically significant. LN, Lymph node; POPDC3, popeye domain-containing protein 3 .

cut-off value of POPDC3 were categorized as low (0.8 to 5.7), intermediate (5.7 to 8.2) and high (8.2 to 11.0) based on OS information (Fig. 5).

To evaluate the prognostic value of POPDC 3 in patients with HNSCC, the 161 patient samples were divided into two groups: Low POPDC 3 expression group $(n=81)$ and high POPDC 3 expression group $(n=80)$ based on the median value. A $\chi^{2}$ test showed that elevated POPDC 3 expression in primary tumors was significantly associated with sex (male; $\mathrm{P}<0.001)$ and age $\geq 60(\mathrm{P}<0.001$; Table III). Univariate analysis showed that higher POPDC 3 expression levels and lymph node metastases predicted poorer survival [hazard ratio $(\mathrm{HR})=1.886$, CI 1.019-3.491; $\mathrm{P}=0.043$ ] and a HR=2.309 (CI 1.163-4.584; $\mathrm{P}=0.017$ ), respectively. Multivariate Cox proportional hazard analyses were used to control for potential confounding variables. The multivariate Cox analysis showed that POPDC3 expression levels ( $\mathrm{HR}=0.516, \mathrm{CI}, 0.270-0.985 ; \mathrm{P}=0.045)$ and lymph node metastasis $(\mathrm{HR}=2.456, \mathrm{CI}, 1.088=5.546 ; \mathrm{P}=0.031$ ) were independent prognostic factors in patients with HNSCC (Table IV).

Construction and validation of the OS nomograms. The significant influencing factors, POPDC3 expression levels, lymph node metastasis and age at diagnosis were incorporated to create the prognostic nomograms for estimating the 3 - and 5 -year OS of patients with HNSCC (Fig. 6A). By adding up these scores to the total on the bottom scale, the 3- and 5-year OS of patients with HNSCC could be predicted. Prognostic nomogram validation was conducted using receiver operating characteristic (ROC) curves (Fig. 6B). The ROC curve showed that the area under the curve for nomogram predictions of the 3 - and 5-year survival rates were 0.681 and 0.58 , respectively. These results confirm that the prognostic nomograms were reasonably accurate. The ROC curves demonstrated excellent agreement between actual survival and nomogram prediction. Based on the prognostic factors of an individual patient with HNSCC, it was possible to obtain a score associated with each prognostic factor on the nomogram point scale and calculate the total score. From this it was possible to evaluate the 3 - and 5 -year survival rates by projecting the total points to the total score scale of the nomogram.

\section{Discussion}

HNSCC remains a fatal malignancy with a $\sim 60 \% 5$-year OS rate for all stages combined (1). Radiation therapy remains one of the most effective treatment options for HNSCC. However, locoregional failure is the most common cause of death, suggesting the presence of a radioresistant subpopulation of cells. Therefore, local disease recurrence requires further investigation $(18,19)$. The previously irradiated exposure area does not benefit from re-irradiation due to radiation resistance of the cancer cells (20). Therefore, further investigations of the mechanisms leading to the unresponsiveness to radiation therapy and the ability to better predict locoregional relapses are urgently required. Additional knowledge regarding radioresistant-associated molecular perturbations in HNSCC may assist in the development of novel strategies to overcome HNSCC radioresistance.

At present, few studies have analyzed GEO databases to identify potential differences in transcriptional regulation and the network of proteins involved in radioresistance of HNSCC. In the present study, the gene expression data from the GEO database (GSE9716) was downloaded and divided into non-irradiated and post-irradiation groups. A comprehensive analysis based on the combination of DEGs and WGCNA was performed to identify valuable potential prognostic factors and biomarkers. The DEGs that exhibited higher expression levels the in radioresistant samples compared the radiosensitive samples were mapped. A total of 86 DEGs, including 77 upregulated genes and 9 downregulated genes were identified 

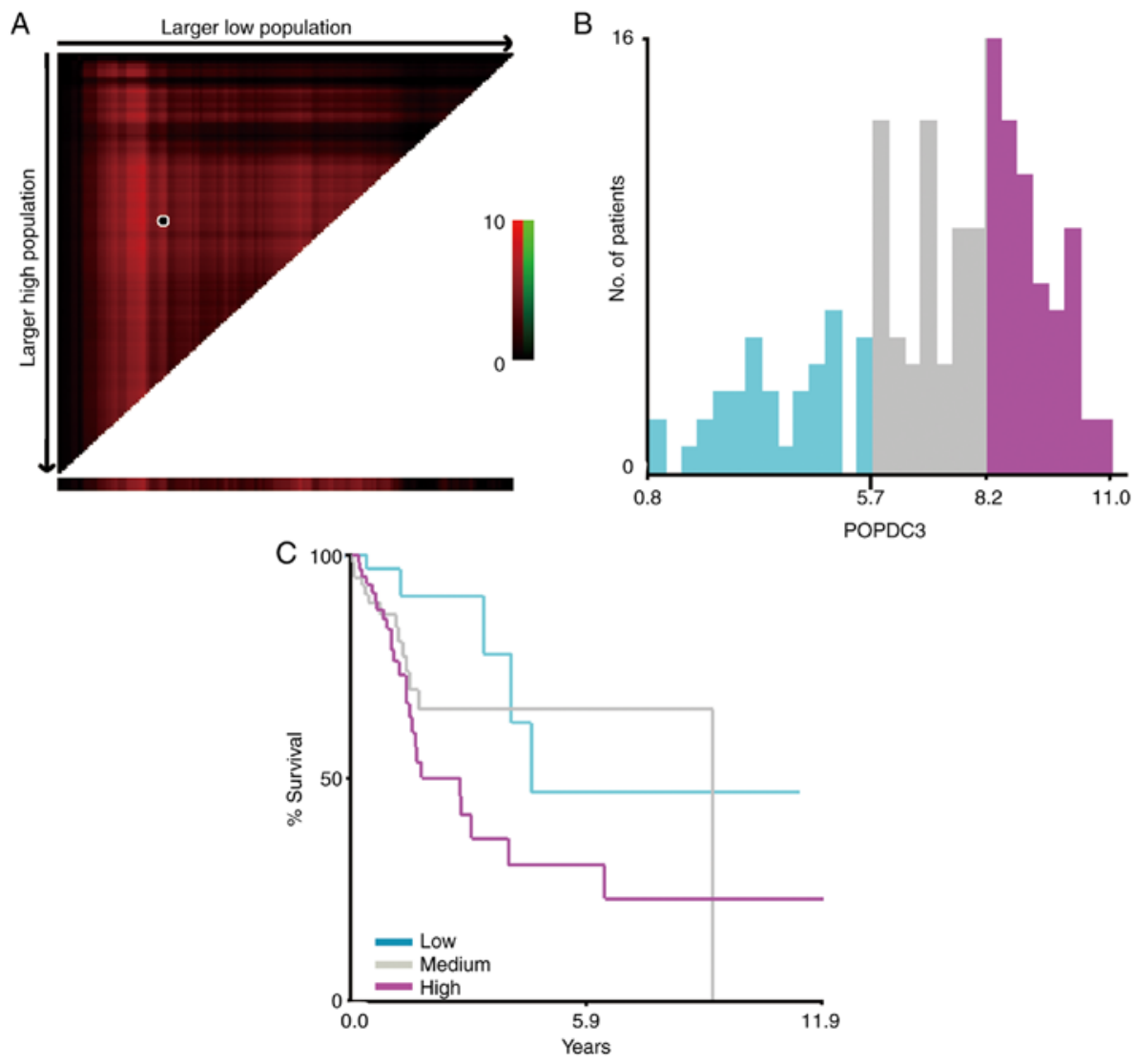

Figure 5. Identification of the optimal cut-off values of POPDC3 for X-tile analysis of The Cancer Genome Atlas cohort. (A) The black dot indicates that optimal cut-off values of POPDC3 had been identified. (B) A histogram and (C) Kaplan-Meier analysis based on the identified POPDC3 cut-off values. Optimal cut-off values of POPDC3 were identified as 5.7 and 8.2 based on overall survival. POPDC3, popeye domain-containing protein 3.

\section{A Nomogram for overall survival}

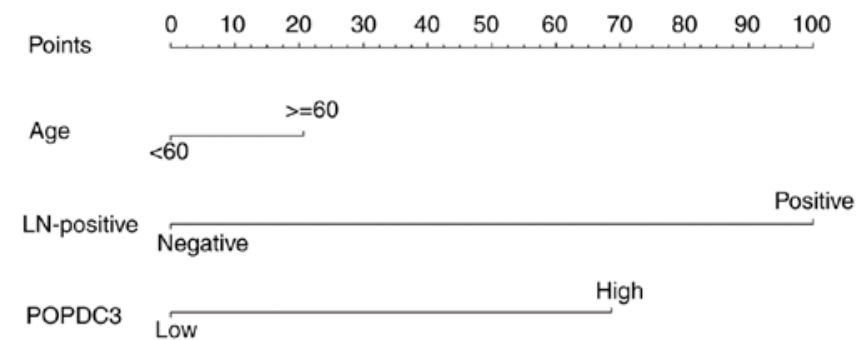

Total points

$$
\begin{array}{llllllllllll}
0 & 20 & 40 & 60 & 80 & 100 & 120 & 140 & 160 & 180 & 200 & 220
\end{array}
$$
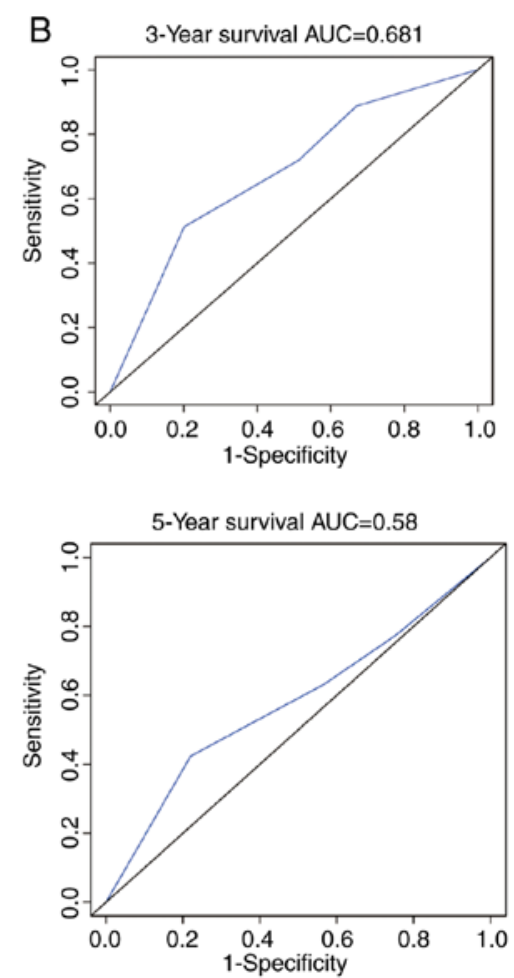

Figure 6. A nomogram to predict survival risk based on POPDC3 and other factors in patients with head and neck squamous cell carcinoma. (A) Evaluation of nomogram-integrated age, POPDC 3 and lymph node biopsy positive for predicting the 3 - and 5-year overall survival. Points of each variable were acquired by drawing a vertical line between each variable and points on the scale. Predicted 3- and 5-year survival rate was calculated according to the total points of each variable by drawing a vertical line from the total points scale to the overall survival scale. (B) Predicting the accuracy of the nomogram based on age, POPDC 3 and lymph node biopsy positive using receiver operating characteristic curves. POPDC3, popeye domain-containing protein 3; AUC, area under the curve. 
Table IV. Multivariate cox regression analysis for overall survival in head and neck squamous cell carcinoma patients.

\begin{tabular}{|c|c|c|c|c|}
\hline \multirow[b]{2}{*}{ Variables } & \multicolumn{2}{|c|}{ Univariate analysis } & \multicolumn{2}{|c|}{ Multivariate analysis } \\
\hline & $\mathrm{HR}(95 \% \mathrm{CI})$ & $\mathrm{P}$ & $\mathrm{HR}(95 \% \mathrm{CI})$ & $\mathrm{P}$ \\
\hline POPDC3 (High vs. Low) & $1.886(1.019-3.491)$ & $0.043^{\mathrm{a}}$ & $0.516(0.270-0.985)$ & $0.045^{\mathrm{a}}$ \\
\hline Sex (Female vs. Male) & $0.989(0.488-2.007)$ & 0.976 & $1.178(0.606-1.679)$ & 0.535 \\
\hline Age ( $\geq 60$ vs. 60 ) & $1.082(0.589-1.990)$ & 0.799 & $1.319(0.691-2.519)$ & 0.401 \\
\hline T stage & & & $0.738(0.474-1.149)$ & 0.179 \\
\hline $\mathrm{T} 1$ & $\mathrm{RV}$ & & & \\
\hline $\mathrm{T} 2$ & $0.647(0.166-2.516)$ & 0.530 & & \\
\hline $\mathrm{T} 3$ & $0.883(0.250-3.117)$ & 0.847 & & \\
\hline $\mathrm{T} 4$ & $0.510(0.151-1.722)$ & 0.278 & & \\
\hline N_stage & & & $1.064(0.658-1.720)$ & 0.801 \\
\hline No & RV & & & \\
\hline N1 & $1.670(0.802-3.480)$ & 0.557 & & \\
\hline $\mathrm{N} 2-\mathrm{N} 3$ & $1.532(0.753-3.120)$ & 0.539 & & \\
\hline Grade & & & $0.738(0.428-1.273)$ & 0.275 \\
\hline G1 & RV & & & \\
\hline $\mathrm{G} 2$ & $1.781(0.539-5.882)$ & 0.344 & & \\
\hline $\mathrm{G} 3$ & $1.290(0.361-4.618)$ & 0.695 & & \\
\hline Clinical_stage & & & $0.994(0.495-1.995)$ & 0.275 \\
\hline $\mathrm{I}+\mathrm{II}$ & $\mathrm{RV}$ & & & \\
\hline III & $1.886(0.602-5.905)$ & 0.276 & & \\
\hline IV & $1.222(0.426-3.511)$ & 0.709 & & \\
\hline LN_positive & & & $2.456(1.088-5.546)$ & $0.031^{\mathrm{a}}$ \\
\hline Negative & $\mathrm{RV}$ & & & \\
\hline Positive & $2.309(1.163-4.584)$ & $0.017^{\mathrm{a}}$ & & \\
\hline LN_invasion & & & $1.178(0.606-2.290)$ & 0.628 \\
\hline Negative & $\mathrm{RV}$ & & & \\
\hline Positive & $1.363(0.748-2.484)$ & 0.311 & & \\
\hline
\end{tabular}

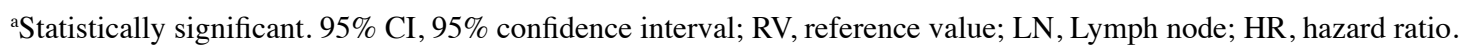

in the non-irradiation group, while a total of 405 DEGs, including 385 upregulated genes and 20 downregulated genes were identified in the post-irradiation group. GO term enrichment and KEGG pathway analysis were used to further investigate the functional enrichment of these two groups of genes. In WGCNA, the light green module in the non-irradiation group and the blue module in the post-irradiation group were the highest correlative modules to radioresistance traits. The genes in these two modules were further selected for hub genes with a cut-off correlation of $>0.8$. A total of 76 and 917 hub genes were selected in the two groups, respectively. The overlap between DEGs and hub genes from the two groups yielded a map of 13 shared DEGs. The 13 genes were screened as candidate key genes; genes which may function to maintain the radioresistant characteristics of cancer cells and promote the radioresistant abilities of cancer cells post-irradiation. According to the FC of the expression of the $13 \mathrm{key}$ genes in the post-irradiation group relative to the non-irradiation group, PBK and POPDC3 were the top two high expression genes in the post-irradiation group compared with the non-irradiation group. Therefore, PBK and POPDC3 were selected for survival analysis. Increased expression of POPDC3 was associated with a poorer survival rate in patients with HNSCC.

POPDC3 is a member of the popeye domain-containing family which consists of three members (21-23). As a second messenger molecule, cAMP participates in numerous cellular functions, physiologically and pathophysiologically $(24,25)$. The POPDC family of proteins are a class of cAMP-binding proteins and have been reported to be involved in cancer progression and associated with prognosis in patients with cancer. Gene expression profiling has shown POPDC1 in breast carcinoma $(26,27)$, colon cancer (26) and gastric cancer (28). POPDC2 upregulation enhances arsenic trioxide-mediated breast cancer cell apoptosis (29), and low expression of POPDC3 is correlated with colorectal cancer drug chemoradiotherapy (21). Hypermethylation of the POPDC3 promoter region is associated with poor survival in patients with gastric cancer (30,31). Luo et al (31) hypothesized that POPDC3 may serve a role in cell adhesion, cell motility, DNA methylation 
and tumorigenesis signaling pathways (31). In the present study, KEGG pathway enrichment showed that from the DEGs in the post-irradiated group, POPDC3 participated in the majority of the biologically active signaling pathways, including viral carcinogenesis, activation of MAPK activity, positive regulation of $\kappa \mathrm{B}$ kinase/NF- $\mathrm{B}$ signaling pathway and others. However, the exact role of POPDC 3 in HNSCC pathogenesis, its role in radioresistance and the underlying molecular mechanisms remain unknown.

To the best of our knowledge, the present study is the first to study examine both the overexpression profiles of POPDC3 in patients HNSCC and in radioresistant HNSCC compared with their respective counterparts, by analyzing both the GEO and Oncomine databases. POPDC3 was upregulated in radioresistant esophageal and lung cancer, further highlighting the potential role of POPDC3 in the development of radioresistance. Kaplan-Meier analysis also indicated that high expression of POPDC3 was associated with poor OS in patients with HNSCC. Collectively, the results of the present study demonstrated that high expression of POPDC3 is associated with radioresistance and poor prognosis in patients with HNSCC. Therefore, POPDC3 may serve as a novel predictive biomarker for HNSCC prognosis and radioresistance in the diagnosis and treatment of patients with HNSCC. However, due to the limitation of the sample size of GSE9716 dataset, a larger scale investigation of the role of POPDC3 involved in the HNSCC development is recommended.

Using TCGA cohort and X-tile analysis, it was demonstrated that high expression of POPDC3 predicted a poor clinical outcome $(\mathrm{P}<0.05)$. Using $\mathrm{X}$-tile analysis, the optimal cut-off value of POPDC3 were categorized as low (0.8 to 5.7), intermediate (5.7 to 8.2) and high (8.2 to 11.0) based on OS information.

Based on TCGA data, sufficient cases were obtained to develop and validate nomograms to predict 3- and 5-year OS in patients with HNSCC. To accurately select prognostic factors, univariate log-rank and multivariate Cox analysis were used to identify independent prognostic factors. The results showed that POPDC3 expression levels and lymph node metastasis were independent prognostic factors for the survival of patients with HNSCC.

Although there is still inadequate evidence to support POPDC3 as an independent prognostic marker for HNSCC, the nomogram model based on POPDC3 expression levels provided a perfect prognostic value and the ROC curve for the nomogram showed a good fit at 3- and 5-year follow-ups. The area under the curve of the ROC curves for nomogram predictions of the 3 - and 5-year survival rates were 0.681 and 0.58 , suggesting a good predictive ability for patients with HNSCC, suggesting that the model was accurate and may possess potential to be applied clinically. Furthermore, a number of assays in vitro and in vivo are required to investigate the effect of modulating POPDC3 expression levels on HNSCC cell lines and the molecular mechanisms underlying POPDC3-mediated regulation of radioresistance in HNSCC need further study.

In conclusion, the aim of the present study was to identify predictive biomarkers predictive for radioresistance in patients with HNSCC by mining gene expression data. The transcriptional profiling followed by bioinformatics analysis processing of radioresistant and radiosensitive samples identified POPDC3 in radioresistance in patients with HNSCC, and also identified POPDC3 expression as a novel biomarker for poor response to radiotherapy. This may highlight POPDC3 as a potential therapeutic target for radioresistant HNSCC treatment. In addition, nomograms were developed and validated to predict the 3- and 5-year OS of patients with HNSCC based on a population-based cohort database. Using only basic information and POPDC3 expression levels, the nomograms demonstrated a high degree of predictive accuracy. The results of the present study demonstrate that bioinformatics analyses may be useful for identifying predictive biomarkers and that these methods can be utilized to establish transcriptional profiles of radioresistance in other types of cancer.

\section{Acknowledgements}

Not applicable.

\section{Funding}

The present was supported by the National Key Research and Development Program of China (grant no. 2017YFA0205200), the National Natural Science Foundation of China (grant nos.81571785and81771957), and theNaturalScienceFoundation of Guangdong Province, China (grant nos. 2016A030311055, 2016A030313770 and 2018A030313074).

\section{Availability of data and materials}

The datasets used during the present study are available from the corresponding author upon reasonable request.

\section{Authors' contributions}

LL and LT conceived and designed the study and critically revised the manuscript. XH and HX designed and analyzed the data and was a major contributor in writing the manuscript. WZ, MZ, YL and HL gave advice on the study and contributed to the statistics and analysis. All authors have read and approved the manuscript.

\section{Ethics approval and consent to participate}

Not applicable.

\section{Patient consent for publication}

Not applicable.

\section{Competing interests}

The authors declare that they have no competing interests.

\section{References}

1. Siegel RL, Miller KD and Jemal A: Cancer statistics, 2018. CA Cancer J Clin 68: 7-30, 2018.

2. Agostini LP, Stur E, Garcia FM, Ventorim DP, Dos Reis RS, Dettogni RS, Dos Santos EVW, Peterle GT, Maia LL, Mendes SO, et al: ATM, BCL2, and TGF $\beta$ gene polymorphisms as radiotherapy outcome biomarkers in head and neck squamous cell carcinoma patients. Genet Test Mol Biomarkers 21: 727-735, 2017. 
3. Edgar R, Domrachev M and Lash AE: Gene expression omnibus: NCBI gene expression and hybridization array data repository. Nucleic Acids Res 30: 207-210, 2002.

4. Cheng J, Lu X, Wang J, Zhang H, Duan P and Li C: Interactome analysis of gene expression profiles of cervical cancer reveals dysregulated mitotic gene clusters. Am J Transl Res 9: 3048-3059, 2017.

5. Zhu L, Shu Z and Sun X: Bioinformatic analysis of four miRNAs relevant to metastasis-regulated processes in endometrial carcinoma. Cancer Manag Res 10: 2337-2346, 2018.

6. Dennis G Jr, Sherman BT, Hosack DA, Yang J, Gao W, Lane HC and Lempicki RA: DAVID: Database for annotation, visualization, and integrated discovery. Genome Biol 4: P3, 2003.

7. Yu G, Wang LG, Han Y and He QY: clusterProfiler: an R package for comparing biological themes among gene clusters. OMICS 16: 284-287, 2012.

8. Colaprico A, Silva TC, Olsen C, Garofano L, Cava C, Garolini D, Sabedot TS, Malta TM, Pagnotta SM, Castiglioni I, et al: TCGAbiolinks: An R/Bioconductor package for integrative analysis of TCGA data. Nucleic Acids Res 44: e71, 2016.

9. Langfelder $P$ and Horvath S: WGCNA: An R package for weighted correlation network analysis. BMC Bioinformatics 9: $559,2008$.

10. Khodarev NN, Minn AJ, Efimova EV, Darga TE, Labay E, Beckett M, Mauceri HJ, Roizman B and Weichselbaum RR Signal transducer and activator of transcription 1 regulates both cytotoxic and prosurvival functions in tumor cells. Cancer Res 67: 9214-9220, 2007.

11. Xiong W, Zhao J, Yu H, Li X, Sun S, Li Y, Xia Q, Zhang C, $\mathrm{He}$ Q, Gao X, et al: Elevated expression of AKR1C3 increases resistance of cancer cells to ionizing radiation via modulation of oxidative stress. PLoS One 9: e111911, 2014.

12. Gautier L, Cope L, Bolstad BM and Irizarry RA: Affy-analysis of Affymetrix GeneChip data at the probe level. Bioinformatics 20 : 307-315, 2004.

13. Bolstad BM, Irizarry RA, Astrand M and Speed TP: A comparison of normalization methods for high density oligonucleotide array data based on variance and bias. Bioinformatics 19 : 185-193, 2003.

14. Ritchie ME, Phipson B, Wu D, Hu Y, Law CW, Shi W and Smyth GK: limma powers differential expression analyses for RNA-sequencing and microarray studies. Nucleic Acids Res 43: e47, 2015.

15. Horvath S and Dong J: Geometric interpretation of gene coexpression network analysis. PLoS Comput Biol 4: e1000117, 2008.

16. Albert R: Scale-free networks in cell biology. J Cell Sci 118 4947-4957, 2005

17. Seo CH, Kim JR, Kim MS and Cho KH: Hub genes with positive feedbacks function as master switches in developmental gene regulatory networks. Bioinformatics 25: 1898-1904, 2009

18. Schmitz S, Ang KK, Vermorken J, Haddad R, Suarez C, Wolf GT Hamoir M and Machiels JP: Targeted therapies for squamous cell carcinoma of the head and neck: Current knowledge and future directions. Cancer Treat Rev 40: 390-404, 2014.

19. Zibelman M and Mehra R: Overview of current treatment options and investigational targeted therapies for locally advanced squamous cell carcinoma of the head and neck. Am J Clin Oncol 39: 396-406, 2016
20. Corvò R: Evidence-based radiation oncology in head and neck squamous cell carcinoma. Radiother Oncol 85: 156-170, 2007.

21. Brand T and Schindler R: New kids on the block: The Popeye domain containing (POPDC) protein family acting as a novel class of cAMP effector proteins in striated muscle. Cell Signal 40 156-165, 2017.

22. Amunjela JN and Tucker SJ: POPDC proteins as potential novel therapeutic targets in cancer. Drug Discov Today 21: 1920-1927, 2016.

23. Andrée B, Hillemann T, Kessler-Icekson G, Schmitt-John T, Jockusch $\mathrm{H}$, Arnold $\mathrm{HH}$ and Brand T: Isolation and characterization of the novel popeye gene family expressed in skeletal muscle and heart. Dev Biol 223: 371-382, 2000.

24. Plattner F, Hayashi K, Hernández A, Benavides DR, Tassin TC, Tan C, Day J, Fina MW, Yuen EY, Yan Z, et al: The role of ventral striatal cAMP signaling in stress-induced behaviors. Nat Neurosci 18: 1094-1100, 2015.

25. Kumar N, Prasad P, Jash E, Jayasundar S, Singh I, Alam N, Murmu N, Somashekhar SP, Goldman A and Sehrawat S: cAMP regulated $\mathrm{EPACl}$ supports microvascular density, angiogenic and metastatic properties in a model of triple negative breast cancer. Carcinogenesis 39: 1245-1253, 2018.

26. Williams CS, Zhang B, Smith JJ, Jayagopal A, Barrett CW, Pino C, Russ P, Presley SH, Peng D, Rosenblatt DO, et al: BVES regulates EMT in human corneal and colon cancer cells and is silenced via promoter methylation in human colorectal carcinoma. J Clin Invest 121: 4056-4069, 2011.

27. Amunjela JN and Tucker SJ: POPDC1 is suppressed in human breast cancer tissues and is negatively regulated by EGFR in breast cancer cell lines. Cancer Lett 406: 81-92, 2017.

28. Kim M, Jang HR, Haam K, Kang TW, Kim JH, Kim SY, Noh SM, Song KS, Cho JS, Jeong HY, et al: Frequent silencing of popeye domain-containing genes, BVES and POPDC3, is associated with promoter hypermethylation in gastric cancer. Carcinogenesis 31: 1685-1693, 2010.

29. Wang X, Gao P, Long M, Lin F, Wei JX, Ren JH, Yan L, He T, Han Y and Zhang HZ: Essential role of cell cycle regulatory genes p21 and p27 expression in inhibition of breast cancer cells by arsenic trioxide. Med Oncol 28: 1225-1254, 2011

30. Spitzner M, Emons G, Kramer F, Gaedcke J, Rave-Fränk M, Scharf JG, Burfeind P, Becker H, Beissbarth T, Ghadimi BM, et al: A gene expression signature for chemoradiosensitivity of colorectal cancer cells. Int J Radiat Oncol Biol Phys 78: 1184-1192, 2010

31. Luo D, Lu ML, Zhao GF, Huang H, Zheng MY, Chang J, Lv L and Luo JB: Reduced Popdc3 expression correlates with high risk and poor survival in patients with gastric cancer. World J Gastroenterol 18: 2423-2429, 2012.

This work is licensed under a Creative Commons Attribution-NonCommercial-NoDerivatives 4.0 International (CC BY-NC-ND 4.0) License. 